\title{
openheart Creatine kinase during non-ST-segment elevation acute coronary syndromes is associated with major bleeding
}

\author{
Lizzy Maritza Brewster (D) , ${ }^{1} \mathrm{Jim}$ Fernand $^{2}$
}

To cite: Brewster LM, Fernand J. Creatine kinase during non-ST-segment elevation acute coronary syndromes is associated with major bleeding. Open Heart 2020;7:e001281. doi:10.1136/ openhrt-2020-001281

Received 29 February 2020 Revised 2 November 2020 Accepted 3 November 2020

Check for updates

(c) Author(s) (or their employer(s)) 2020. Re-use permitted under CC BY-NC. No commercial re-use. See rights and permissions. Published by BMJ.

${ }^{1}$ Department of Cardiovascular Disease, CK Science Foundation, Amsterdam, The Netherlands ${ }^{2} \mathrm{Clinic}$ for Health and Individual Medicine, Utrecht, Netherlands

Correspondence to Dr Lizzy Maritza Brewster; mail@lizzybrewster.net

\begin{abstract}
Background It was recently reported that highly elevated plasma activity of the ADP-scavenging enzyme creatine kinase (CK), to $>10$ times the upper reference limit (URL), is independently associated with fatal or non-fatal bleeding during treatment for ST-segment elevation myocardial infarction (OR $2.6(95 \% \mathrm{Cl}, 1.8$ to 2.7)/log CK increase). Evidence indicates that CK attenuates ADP-dependent platelet aggregation. This study investigates whether moderately elevated CK in non-ST-segment elevation acute coronary syndrome (NSTE-ACS) is associated with major bleeding. Methods The Thrombolysis In Myocardial Ischemia (TIMI) 3B trial compared recombinant tissue-type plasminogen activator (rt-PA) (35-80 mg) with placebo and early catheterisation with conservative management in patients with NSTE-ACS. Main outcomes of the current study are the independent association of peak plasma CK (CKmax) with adjudicated fatal or non-fatal major bleeding (primary) and with combined major bleeding, stroke and hospital death (secondary), with covariables including age, sex, body mass index, systolic blood pressure, creatinine and assignment to add-on rt-PA versus placebo. Discrimination was assessed with C-statistics.
\end{abstract}

Results The study included 1473 patients $(66 \%$ men, $80 \%$ white, mean age 59 years, SE 0.3). CKmax ranged between 15 and 19045 IU/L (mean (SE), 450 (24) IU/L; two times URL). Major bleeding occurred in $2.0 \%$ (mean age 65 (1.3) years; mean CKmax 1015 (319) IU/L; six times URL), and the combined outcome in $4.3 \%$ of the patients, adjusted OR per log CK increase, respectively, 3.1 (1.6 to 5.9) for major bleeding and 3.9 (2.5 to 6.1) for the combined outcome; $\mathrm{C}$-index 0.8 for both outcomes. The association between CK and bleeding was independent of the use of thrombolytic therapy.

Discussion The presented data add to the existing evidence that proportionate to its plasma activity, the ADP-binding enzyme CK is strongly and independently associated with non-fatal and fatal major bleeding during treatment for NSTE-ACS. CK might increase the accuracy of prediction models for major bleeding in patients with NSTE-ACS.

Trial registration number NCT00000472.

\section{BACKGROUND}

Major bleeding is the most common noncardiac cause of mortality during treatment

\section{Key questions}

What is already known about this subject?

- The development of increasingly potent antithrombotic drugs for acute coronary syndrome (ACS) prompts new, more precise approaches to assess the individual risk for major bleeding. The ADPscavenging enzyme creatine kinase (CK) inhibits ADP-dependent platelet aggregation in vitro and was recently reported to be independently associated with bleeding during treatment for ST-segment elevation myocardial infarction (adjusted OR for fatal/non-fatal bleeding $2.6(95 \% \mathrm{Cl}, 1.8$ to 3.7$)$ per log CK increase).

What does this study add?

- Peak CK during treatment for non-ST-segment elevation acute coronary syndrome (NSTE-ACS) is strongly and independently associated with nonfatal or fatal major bleeding and with combined major bleeding, stroke or hospital death (adjusted OR, respectively, 3.1 (1.6 to 5.9 ) and 3.9 (2.5 to 6.1 ) per $\log$ CK increase).

How might this impact on clinical practice?

- Obsolescent CK diagnostics may help identify patients at risk for major bleeding during treatment for NSTE-ACS.

of non-ST-segment elevation acute coronary syndrome (NSTE-ACS). Therefore, it is important to identify patients at risk of haemorrhagic complications during the management of ACS. ${ }^{1-4}$ Evidence indicates that the ADP-binding enzyme creatine kinase (CK) inhibits ADP-dependent platelet activation and subsequent aggregation (figure 1). ${ }^{5-8}$ During myocardial ischaemia and infarction, CK and other cell constituents such as myoglobin and troponin enter plasma along with smaller molecules including phosphocreatine ${ }^{6-8}$ Extracellular CK is thought to reduce ADP and ADP-dependent platelet activation through its scavenging action on ADP or through conversion into ATP, catalysing the reaction ${ }^{6-8}$ :

ADP + Phosphocreatine $<=>$ ATP + Creatine 


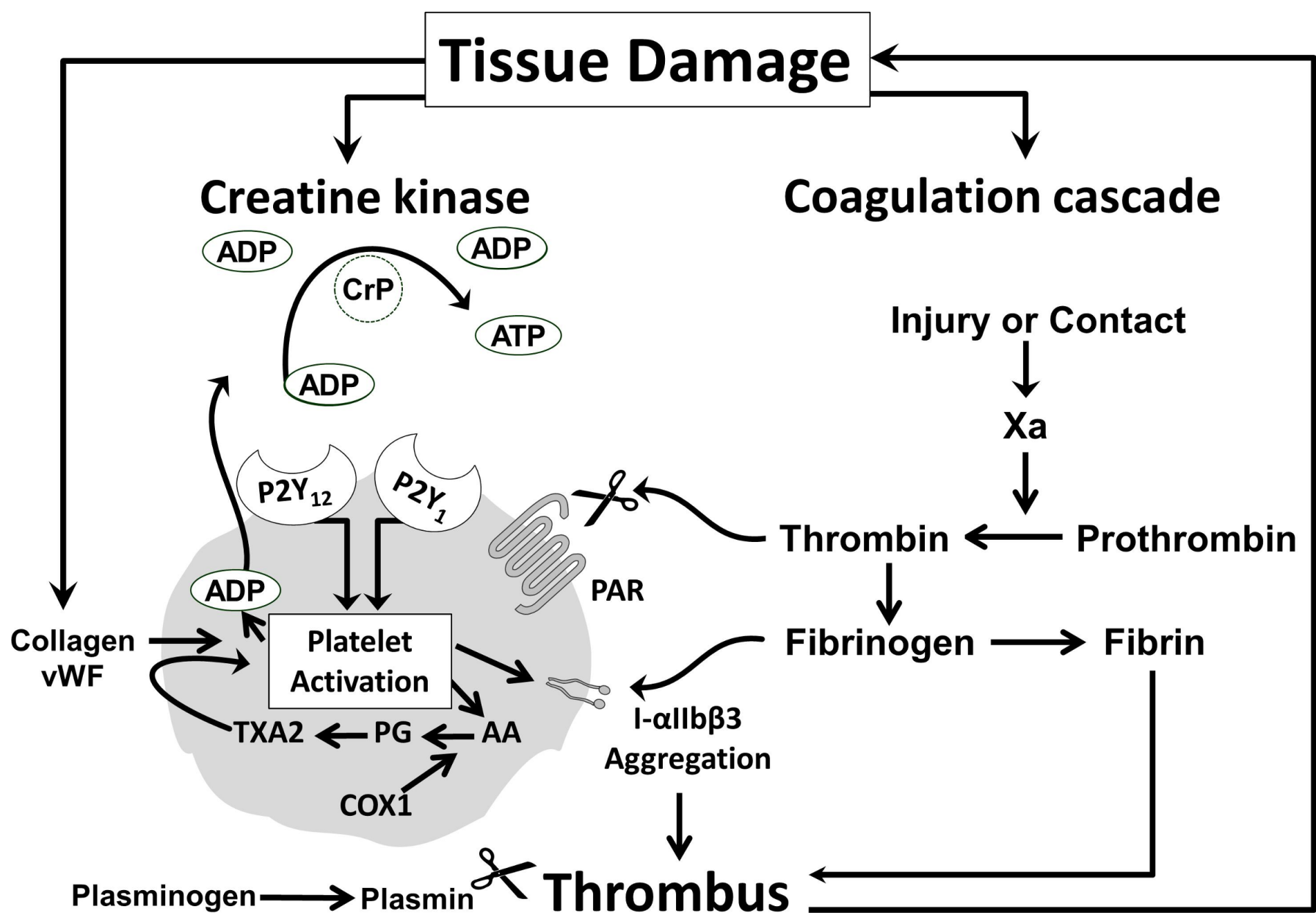

Figure 1 The ADP-binding enzyme creatine kinase $(C K)$ and inhibition of platelet activation. Central to the development of acute coronary syndrome (ACS) is plaque rupture or erosion resulting in platelet activation, aggregation and thrombosis. ${ }^{1-5}$ CK was recently proposed to be an essential part of the counterregulatory mechanism of endothelial thromboresistance that limits platelet activation. ${ }^{67}$ After plaque ruptures, platelets adhere to the injured vessel wall and undergo activation through collagen and von Willebrand factor (VWF). Activated platelets release substances including ADP and thromboxane A2 (TXA2) synthesised from arachidonic acid (AA) through prostaglandin (PG) catalysed by COX1. ADP and TXA2 amplify the response to injury and produce sustained platelet aggregation. Furthermore, thrombin generated by tissue damage activates platelets' protease activated receptors (PAR), leading to the generation of more thrombin on the platelets' surface, further platelet activation, conversion of fibrinogen to fibrin and further stabilisation of the platelet-fibrin clot. Fibrin is degraded by plasmin, formed by plasminogen and (recombinant) tissue-type plasminogen activator. ADP is considered to be central to platelet activation. ADP stimulation of the P2Y1 receptor activates phospholipase $\mathrm{C}$ resulting in weak, transient platelet aggregation. Activation of P2Y12 receptor results in the activation of glycoprotein receptors Ilb/Illa (integrin (I)- $\alpha$ llb $\beta 3$ ) and firm platelet aggregation. ${ }^{56} \mathrm{CK}$ strongly binds ADP and reduces platelet aggregation in the presence of phosphocreatine $(\mathrm{CrP}) .{ }^{78} \mathrm{The}$ enzyme might act in synergy with antithrombotic or fibrinolytic drugs that target factor Xa, thrombin, PAR, COX1, P2Y12, $\mathrm{I}-\alpha \mathrm{llb} \beta 3$, plasminogen or other factors ${ }^{12}$ to increase bleeding risk in ACS $^{6}$ (modified after Brewster and Fernand, 2020). ${ }^{6}$

In line with this, it was recently reported that peak plasma CK (CKmax) during admission is strongly and independently associated with bleeding after ST-segment elevation myocardial infarction (STEMI), with an adjusted OR per log CK increase of 2.6 (95\% CI, 1.8 to 3.7$)$ for fatal/non-fatal bleeding and 3.1 (2.2 to 4.4) for bleeding/all-cause mortality. ${ }^{6}$ As the effect of CK on ADP-dependent platelet aggregation is thought to act in synergy with thrombolytic or antithrombotic (antiplatelet or anticoagulant) therapy, ${ }^{6}$ lower CK levels might also be associated with bleeding. Therefore, it is studied whether moderately elevated CK during NSTE-ACS is independently predictive of major bleeding.

\section{METHODS}

The TIMI 3 trial

Data of the Thrombolysis In Myocardial Ischemia 3 (TIMI 3) trial where CK was routinely estimated during admission in all patients were used. ${ }^{910}$ As reported previously, ${ }^{910}$ the TIMI 3 study consisted of two separate multicentre, randomised controlled double-blind clinical trials designed to assess the effects of recombinant tissue-type plasminogen activator (rt-PA) versus placebo added to conventional therapy 
Interventional cardiology

Table 1 Patients' characteristics

\begin{tabular}{|c|c|c|c|}
\hline \multirow[b]{2}{*}{ Clinical parameters } & \multicolumn{3}{|l|}{ Bleeding } \\
\hline & None & Non-major & Adj. major \\
\hline $\mathrm{N}(\%)$ & $1345(91.3)$ & $98(6.7)$ & $30(2.0)$ \\
\hline Men $(\%)$ & 67 & 57 & 70 \\
\hline Age (years) & $58(0.3)$ & $61(0.1)$ & $65(1.3)$ \\
\hline Ancestry (white,\%) & 79 & 90 & 80 \\
\hline $\mathrm{BMI}\left(\mathrm{kg} / \mathrm{m}^{2}\right)$ & $28(0.1)$ & $27(0.5)$ & $27(0.8)$ \\
\hline H/O Cigarette smoking (\%) & 69 & 68 & 69 \\
\hline H/O Hypertension (\%) & 42 & 43 & 36 \\
\hline H/O Diabetes mellitus (\%) & 15 & 11 & 19 \\
\hline H/O Previous angina pectoris (\%) & 85 & 92 & 87 \\
\hline H/O Myocardial infarction (\%) & 41 & 34 & 21 \\
\hline $\mathrm{FH} / 0 \mathrm{CAD}(\%)$ & 39 & 35 & 23 \\
\hline On diuretics at adm. (\%) & 16 & 20 & 30 \\
\hline Aspirin within 24 hours before adm. (\%) & 48 & 43 & 45 \\
\hline Aspirin dose before adm. (mg/d) & $325(0-325)$ & $325(0-325)$ & $325(0-325)$ \\
\hline R/ Hypercholesterolaemia (\%) & 20 & 15 & 7 \\
\hline $\mathrm{SBP}(\mathrm{mm} \mathrm{Hg})^{\star}$ & $132(0.6)$ & $132(1.8)$ & $129(2.8)$ \\
\hline Platelet count $\left(.10^{9} / \mathrm{L}\right)^{*}$ & $264(2.0)$ & $275(9.2)$ & $269(14.2)$ \\
\hline $\mathrm{HB}(\mathrm{mmol} / \mathrm{L})^{*}$ & $8.8(0.0)$ & $8.6(0.1)$ & $9.3(0.7)$ \\
\hline HT $(\%)^{*}$ & $41.5(0.1)$ & $40.9(0.5)$ & $40.5(1.4)$ \\
\hline aPTT >1.5URL at adm. (\%) & 12 & 11 & 13 \\
\hline WBC count $\left(.10^{9} / \mathrm{L}\right)^{*}$ & 8.7 & 9.0 & 10.6 \\
\hline Creatinine $(\mu \mathrm{mol} / \mathrm{L})^{\star}$ & $97(80-115)$ & $88(80-106)$ & $97(71-117)$ \\
\hline Invasive treatment arm (\%) & 49 & 61 & 62 \\
\hline rt-PA (\%) & 48 & 57 & 73 \\
\hline Fibrinogen 12 hours (g/L) & $2.7(0.0)$ & $2.6(0.1)$ & $2.4(0.1)$ \\
\hline FDP $12 \mathrm{~h} \mathrm{~min} \mathrm{(mg/L)}$ & $0.22(0.11)$ & $0.24(0.24)$ & $1.20(0.8)$ \\
\hline Heparin dose d. 1-5 (U/d) & $17620(169)$ & $16835(565)$ & 15263 (1 402) \\
\hline Aspirin dose d. 1-5 (mg/d) & $240(2.4)$ & $234(10.7)$ & $181(24.4)$ \\
\hline HB d. 1-5 (mmol/L) & $12.9(0.0)$ & $12.0(0.2)$ & $11.6(0.3)$ \\
\hline Platelet count d. $1-5\left(.10^{9} / \mathrm{L}\right)$ & $238(1.9)$ & $242(7.6)$ & $202(12.3)$ \\
\hline CKmax (IU/L) & $149(82-424)$ & $180(83-436)$ & $390(174-1104)$ \\
\hline CKmax (times URL) & $0.7(0.4-2.0)$ & $0.9(0.4-2.0)$ & $1.6(0.9-5.7)$ \\
\hline CKmax <200 IU/L/<URL (\%) & $60 / 59$ & $55 / 53$ & $30 / 40$ \\
\hline Time to peak CK (h) & $4(0-24)$ & $4(12-48)$ & $4(24-72)$ \\
\hline Time to bleeding $(\mathrm{h}) \dagger$ & n.a. & $96(72-120)$ & $72(48-144)$ \\
\hline
\end{tabular}

Patients by adjudicated (adj.), major bleeding (primary outcome) versus other or no bleeding. Data are counts, percentages, mean (SD) or median (IQR), rounded to whole numbers where applicable.

Oral anticoagulant drug use was an exclusion criterion.

${ }^{*}$ At hospital admission (baseline).

†Derived from days.

Adm., admission; aPTT, activated partial thromboplastin time; BMI, body mass index; CAD, coronary artery disease; CKmax, peak plasma creatine kinase during admission; d, day; FDP, fibrin(ogen) degradation products; (F)H/O, (family) history of; h, hour; HB, haemoglobin; HT, haematocrit; n.a, not applicable; R/, drug therapy for; rt-PA, recombinant tissue-type plasminogen activator; SBP, systolic blood pressure; URL, upper reference limit; WBC, white blood cell. 

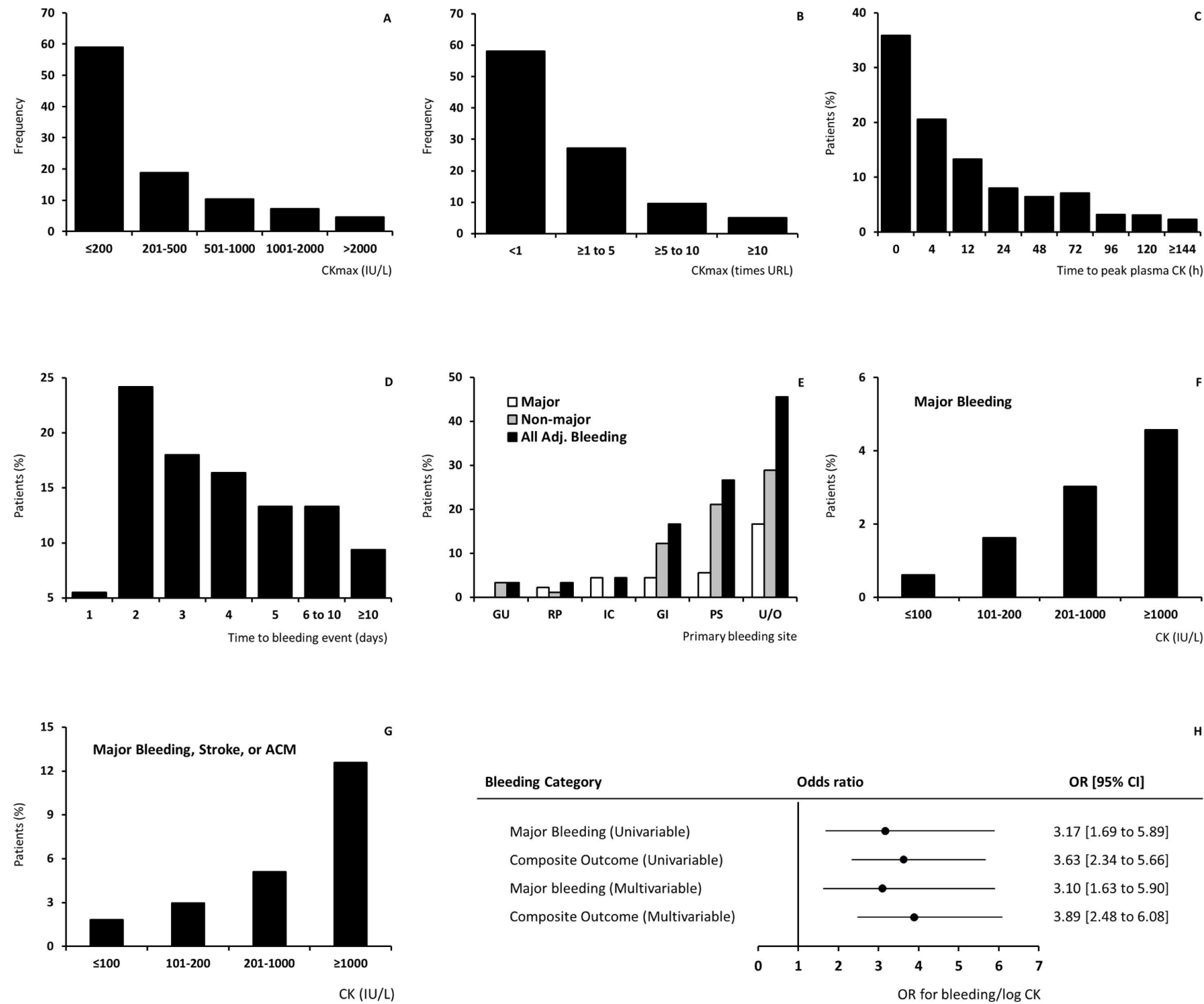

Figure 2 The association between CK and bleeding. (A) Peak plasma creatine kinase (CK) during hospitalisation (IU/L); (B) normalised peak plasma CK activity (times the upper reference limit (URL)); (C) time to peak plasma CK (hours after admission); (D) time to first adjudicated bleeding (days after submission); (E) site and severity of primary adjudicated bleeding; (F) major bleeding by peak plasma CK (patients, \%); (G) major bleeding, stroke or all-cause mortality (ACM) by peak plasma CK (patients, $\%)$; (H) OR for bleeding per log CK increase (point estimate with 95\% CI). Panels (A) (CK, IU/L) and (B) (CK normalised for the upper reference limit (URL)) show CK activity below URL in the majority of patients, as expected with non-ST-segment elevation acute coronary syndromes. Panels (C) and (D) depict the time to peak CK value (day 1) versus time to the first adjudicated bleeding (peak at day 2). Panel (E) shows the site and severity of adjudicated bleeding (GU, RP, IC, GI, PS or U/O are, respectively, genitourinary, retroperitoneal, intracranial, gastrointestinal, puncture site (vascular access site-related) or unknown/ other; with surgical bleeding classified as 'other'); in panels $(\mathrm{F})$ and $(\mathrm{G})$ the primary and secondary outcome by CK level, and in panel $(H)$ the association between log $C K$ adjusted for URL and major bleeding or the composite outcome in univariable and multivariable logistic regression analyses.

(including intravenous heparin and oral aspirin) on the coronary culprit lesion (TIMI 3A, N=391) and on clinical outcomes (TIMI 3B, N=1 473) in comparably selected and treated patients with unstable angina or non-ST-segment elevation myocardial infarction. TIMI 3B further compared management with early cardiac catheterisation (and percutaneous transluminal coronary angioplasty or coronary artery bypass surgery, if appropriate) at 18-48hours after presentation (invasive strategy) versus angiography with revascularisation only if the patient had spontaneous or provokable ischaemia (conservative strategy). The TIMI $3 \mathrm{~A}$ study recruited participants aged $21-76$ years, and TIMI 3B aged 21-79 years, in, respectively, 15 and 25 participating centres across the USA and Canada. All patients had given written informed consent to participate in the study. ${ }^{910}$ Patients were required to have chest discomfort at rest suggestive of myocardial ischaemia, lasting greater than 5 min but less than 6 hours, which occurred within 12 hours (24hours for TIMI 3B) of the time of enrolment. Exclusion criteria were patients who underwent bypass surgery; had 
A. C-index CK and Major Bleeding

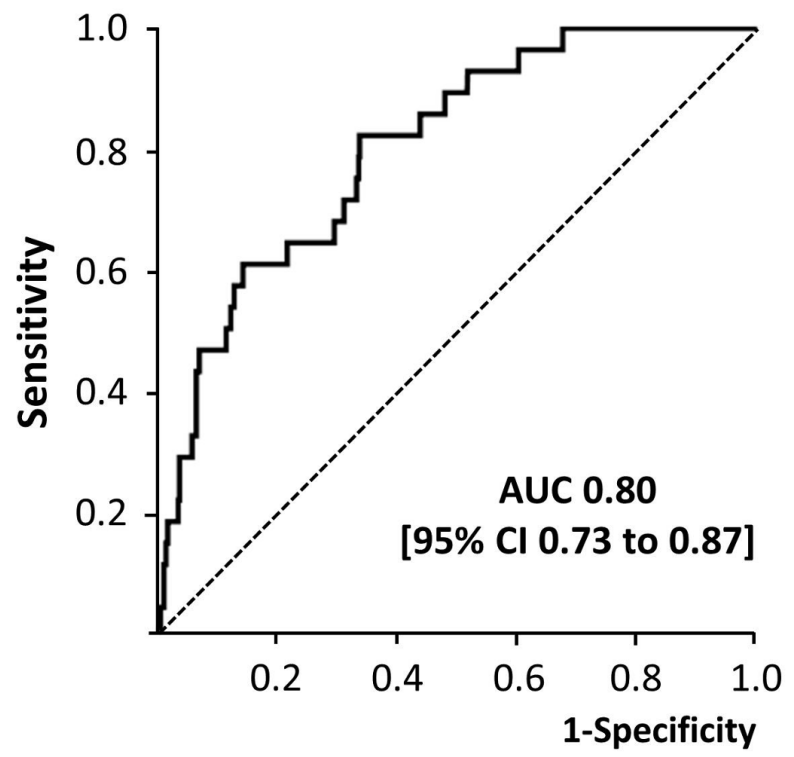

\begin{tabular}{|c|c|c|c|}
\hline Covariables & AUC & {$[95 \%$} & $\% \mathrm{Cll}$ \\
\hline$\overline{C K}$ & 0.68 & {$[0.60$} & $0.76]$ \\
\hline Sex & 0.49 & {$[0.38$} & $0.59]$ \\
\hline Age & 0.68 & {$[0.60$} & $0.76]$ \\
\hline Diabetes Mellitus & 0.51 & {$[0.41$} & $0.62]$ \\
\hline rt-PA & 0.64 & {$[0.54$} & $0.73]$ \\
\hline Diuretics at admission & 0.57 & {$[0.46$} & $0.69]$ \\
\hline MV Model (CK, age, rt-PA) & 0.79 & {$[0.70$} & 0.87 \\
\hline MV Model (CK, age) & 0.80 & {$[0.73$} & $0.87]$ \\
\hline
\end{tabular}

B. C-index CK and Composite Outcome

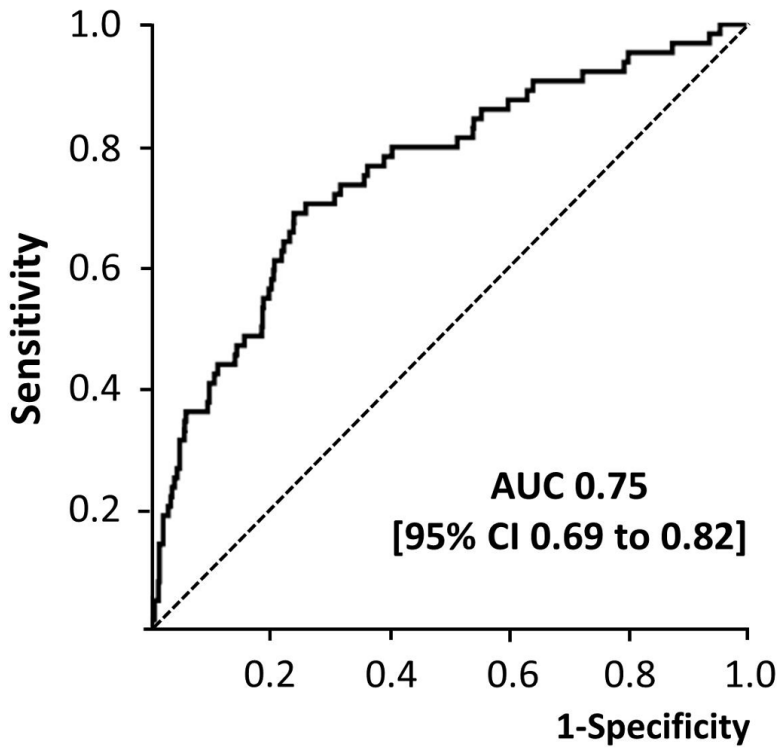

\begin{tabular}{|c|c|c|c|}
\hline Covariables & AUC & \multicolumn{2}{|c|}{$[95 \% \mathrm{Cl}]$} \\
\hline CK & 0.69 & {$[0.62$} & $0.76]$ \\
\hline Sex & 0.53 & {$[0.46$} & $0.61]$ \\
\hline Age & 0.67 & {$[0.60$} & $0.73]$ \\
\hline Diabetes Mellitus & 0.53 & {$[0.45$} & $0.60]$ \\
\hline rt-PA & 0.56 & {$[0.49$} & $0.63]$ \\
\hline Diuretics at admission & 0.52 & {$[0.45$} & $0.60]$ \\
\hline MV M & 0.75 & {$[0.69$} & 0.82 \\
\hline
\end{tabular}

Figure 3 (Panel A) C-index CK and major bleeding. (Panel B) C-index CK and major bleeding, stroke or hospital death. AUC, area under the curve; CK, log creatine kinase; rt-PA, recombinant tissue-type plasminogen activator use; MV, multivariable, sex, women versus men, binomial logistic regression model; parameter units are as in table 1. Bold font indicates statistical significance.

experienced myocardial infarction within the preceding 21 days (excluding within 12 hours before enrolment); had new, persistent ST-segment elevation; had percutaneous transluminal coronary angioplasty in the past 6 months; had contraindication for thrombolytic therapy; used oral anticoagulant therapy; had any history of cerebrovascular disease or transient ischaemic attack, or advanced illness including malignancies, hepatic, renal disorders, among other criteria. Standard clinical care included heparin given as a bolus of $5000 \mathrm{IU}$ intravenously followed by an infusion of $1000 \mathrm{IU} / \mathrm{h}$ to reach activated partial thromboplastin time (aPTT) 1.5-2.0 times normal, and anti-ischaemic therapy with a beta-adrenergic blocker, a calcium channel blocker and a nitrate preparation. Aspirin was not given routinely in TIMI $3 \mathrm{~A}$ and $325 \mathrm{mg}$ daily from day 2 in TIMI 3B. The primary comparison was rt-PA versus placebo (blinded), with a primary endpoint of improvement in perfusion or stenosis in TIMI 3A. In TIMI 3B, the secondary comparison was invasive versus conservative strategy (unblinded), with a combined primary endpoint of reduction in death, (subsequent) myocardial infarction or either spontaneous or provokable ischaemia within or at 6 weeks after study drug infusion. The main secondary evaluation was at day 21 after submission. ${ }^{910}$

\section{Prevention and management of haemorrhagic complications}

Taken the high bleeding rate in the TIMI 2 trial into account (investigator-reported bleeding in $58.4 \%$ of the patients), ${ }^{6} 11$ elaborate measures were taken in TIMI 3 to prevent and treat haemorrhage, such as more stringent contraindications for thrombolytic therapy compared with TIMI 2. ${ }^{6-11}$ While in TIMI $2,100-150 \mathrm{mg}$ rt-PA was used, rt-PA dose was reduced in TIMI 3 to $0.8 \mathrm{mg} / \mathrm{kg}$, with a maximum of $80 \mathrm{mg}$. aPTT-guided heparin dose adjustments were part of the study protocol, and relating to the high number of puncture bleeds in TIMI $2,{ }^{6}{ }^{11}$ heparin 
Table 2 Modelling of the association between CK and major bleeding

\begin{tabular}{|c|c|c|c|c|c|}
\hline Univariable & OR & & & & \\
\hline CK & 3.17 & & & 5.89) & \\
\hline Sex ${ }^{*}$ & 0.84 & & & 1.85) & \\
\hline Age & 1.08 & & & 1.13) & \\
\hline BMI & 0.95 & & & 1.02) & \\
\hline Ancestry† & 0.98 & & & 2.42) & \\
\hline Diabetes mellitus & 1.33 & & & 4.46) & \\
\hline Hypertension & 0.70 & & & 1.50) & \\
\hline Baseline SBP & 1.00 & & & 1.01) & \\
\hline Baseline haematocrit & 0.95 & & & 1.04) & \\
\hline Baseline creatinine & 0.93 & & & 1.07) & \\
\hline rt-PA vs placebo & 3.29 & & & 7.74) & \\
\hline Invasive vs conservative & 1.64 & & & 3.49) & \\
\hline Multivar. (full) & $B$ & SE & Wald & OR & $\mathrm{Cl}$ \\
\hline CK & 1.10 & 0.33 & 11.14 & 3.01 & 1.58 to 5.76 \\
\hline Sex & -0.27 & 0.42 & 0.40 & 0.77 & 0.34 to 1.75 \\
\hline Age & 0.08 & 0.03 & 11.50 & 1.09 & 1.04 to 1.14 \\
\hline Diabetes mellitus & 0.34 & 0.64 & 0.29 & 1.41 & 0.40 to 4.93 \\
\hline rt-PA vs placebo & 1.23 & 0.44 & 7.80 & 3.43 & 1.44 to 8.15 \\
\hline Invasive vs conservative & 0.44 & 0.39 & 1.24 & 1.55 & 0.72 to 3.34 \\
\hline Constant & -10.19 & 1.66 & 37.50 & 0.00 & - \\
\hline \multicolumn{6}{|l|}{ Multivar. (restricted) } \\
\hline CK & 1.13 & 0.33 & 11.92 & 3.10 & 1.63 to 5.90 \\
\hline Ageł & 0.08 & 0.02 & 11.24 & 1.09 & 1.04 to 1.14 \\
\hline rt-PA vs placebo & 1.24 & 0.44 & 7.88 & 3.45 & 1.45 to 8.18 \\
\hline Constant & -9.90 & 1.64 & 36.33 & 0.00 & - \\
\hline
\end{tabular}

Modelling of the association between creatine kinase (CK) and major bleeding.

Parameter units are as in table 1. Baseline platelet count, and fibrinogen and fibrin(ogen) degradation products at 50 min or 12 hours after start therapy were not associated with major bleeding (OR 1; data not shown). Multivar., multivariable full and restricted models. Model parameters full (restricted) model: omnibus test of model coefficients $\chi^{2} 34.2$ (32.4) df 6 (3); -2 log likelihood 251 (253); Bayes information criterion 270 (262) Cox and Snell R square 0.02 (0.02); Nagelkerke R square 0.13 (0.12); Hosmer Lemeshow $\chi^{2} 6.2$ (9.8), df 8 (8); classification overall percentage 98.0 (98.0), $n=1465$ (1465) complete, unimputed cases. Bootstrapping OR was identical to the depicted outcomes (data not shown). Bold font indicates statistical significance.

*Women vs men.

†Non-white vs white.

¥OR age per 10 years (under the assumption of linearity) is 2.27 (1.42 to 3.63). Including diuretic use at admission did not affect the final model. Substituting (log) CKmax during admission with (log) peak CK on day 1 yielded OR 2.64 (1.56 to 4.47) in the final model.

BMI, body mass index; SBP, systolic blood pressure; rt-PA, recombinant tissue-type plasminogen activator.

doses were routinely reduced 4 hours prior to the first arterial puncture for the arteriogram. Major vascular catheter entry sites were minimised and protected with sheath introducers to tamponade the site for approximately 24 hours. Vascular puncture sites were treated with compression dressings, and sites of vascular entry were checked every 2 hours during the acute study period. Other measures included complete blood count at least every 12 hours during the first 24 hours after the intervention and testing of stool, urine and vomitus specimens for occult blood. Frequent clinical assessments and examinations were advised to assess potential internal haemorrhage. If bleeding occurred, management was highly protocolised, and specific therapy was given according to site of bleeding, such as antacids and histamine 2 receptor blockers for upper gastrointestinal bleeding. Finally, life-threatening haemorrhagic complications or any were reported immediately to the Coordinating Centre and the Programme Office. ${ }^{9} 10$

\section{Classification of haemorrhagic events}

Bleeding was classified by the Haemorrhagic Event Review Committee as described previously, ${ }^{11}$ as 'major' when a decrease in haemoglobin of $>50 \mathrm{~g} / \mathrm{L}(>3.1 \mathrm{mmol} / \mathrm{L})$, intracranial bleeding or cardiac tamponade was present, or with death from haemorrhage; as 'minor' if a haemoglobin reduction $30-50 \mathrm{~g} / \mathrm{L}(1.9-3.1 \mathrm{mmol} / \mathrm{L})$ from an identified 
Table 3 Modelling of the association between CK and major bleeding, stroke or death

\begin{tabular}{|c|c|c|c|c|c|}
\hline Univariable & OR & & & & \\
\hline CK & 3.63 & & & $5.66)$ & \\
\hline $\operatorname{Sex}^{\star}$ & 1.27 & & & 2.13) & \\
\hline Age & 1.07 & & & 1.10) & \\
\hline $\mathrm{BMI}$ & 0.95 & & & 1.00) & \\
\hline Ancestry† & 1.33 & & & 2.37) & \\
\hline Diabetes mellitus & 0.57 & & & 1.23) & \\
\hline Hypertension & 0.96 & & & 1.60) & \\
\hline Baseline SBP & 1.00 & & & 1.01) & \\
\hline Baseline haematocrit & 0.99 & & & 1.01) & \\
\hline Baseline creatinine & 0.92 & & & 1.01) & \\
\hline rt-PA vs placebo & 1.59 & & & 2.66) & \\
\hline Invasive vs conservative & 1.53 & & & 2.57) & \\
\hline Multivar. (full) & $\mathrm{B}$ & SE & Wald & OR & $\mathbf{C I}$ \\
\hline CK & 1.31 & 0.23 & 31.89 & 3.71 & 2.35 to 5.85 \\
\hline Sex & 0.14 & 0.28 & 0.25 & 1.15 & 0.67 to 1.99 \\
\hline Age & 0.08 & 0.02 & 20.28 & 1.08 & 1.05 to 1.12 \\
\hline Ancestry & 0.39 & 0.32 & 1.49 & 1.48 & 0.79 to 2.78 \\
\hline Diabetes mellitus & 0.57 & 0.42 & 1.84 & 1.76 & 0.78 to 4.01 \\
\hline rt-PA vs placebo & 0.48 & 0.27 & 3.14 & 1.62 & 0.95 to 2.75 \\
\hline Invasive vs conservative & 0.38 & 0.27 & 1.96 & 1.46 & 0.86 to 2.49 \\
\hline Constant & -8.70 & 1.15 & 57.57 & 0.00 & - \\
\hline \multicolumn{6}{|l|}{ Multivar. (restricted) } \\
\hline CK & 1.36 & 0.23 & 35.38 & 3.89 & 2.48 to 6.08 \\
\hline Age $\neq$ & 0.07 & 0.02 & 20.71 & 1.08 & 1.04 to 1.11 \\
\hline Constant & -7.86 & 1.06 & 55.07 & 0.00 & - \\
\hline
\end{tabular}

Modelling of the association between (log) creatine kinase (CK) and the composite outcome (major bleeding, stroke and death). Parameter units are as in table 1. Baseline platelet count, and fibrinogen and fibrin(ogen) degradation products at 50 min or 12 hours after start therapy were not associated with the composite outcome (OR 1; data not shown). Multivar., multivariable. Model parameters full (restricted) model: omnibus test of model coefficients $\chi^{2} 63.1$ (56.3) df 7 (2); -2 log likelihood 457 (470); Bayes information criterion 479 (476); Cox and Snell R square 0.04 (0.04); Nagelkerke R square 0.14 (0.13); Hosmer Lemeshow $\chi^{2} 6.6$ (10.2), df 8 (8); classification overall percentage 95.7 (95.6), $n=1465$ (1469) complete, unimputed cases. Bootstrapping OR was identical to the depicted outcomes (data not shown). Boldface font indicates statistical significance.

*Women vs men;.

†Non-white vs white.

ҒOR age per 10 years (under the assumption of linearity) is 2.10 (1.53 to 2.87)). Diuretic use at admission did not affect the final model.

Substituting (log) CKmax during admission with (log) peak CK on day 1 yielded OR 2.99 (2.07 to 4.30) for CK in the final model.

$\mathrm{BMI}$, body mass index; rt-PA, recombinant tissue-type plasminogen activator; SBP, systolic blood pressure.

bleeding site was present, or if the patient had spontaneous macroscopic haematuria, haemoptysis or hematemesis; and as 'loss-no-site' if the bleeding site was unknown, but haemoglobin reduction was $40-50 \mathrm{~g} / \mathrm{L}(2.5-3.1 \mathrm{mmol} / \mathrm{L})$. Transfusion of one unit of packed cells or whole blood was counted as $10 \mathrm{~g} / \mathrm{L}$ or haematocrit reduction of $3 \% .^{9-11}$

\section{Laboratory studies}

Total CK activity was estimated at the study centres, collected at baseline (pretreatment), during the first 3 days, at 4, 12, 24, 48 and 72 hours, and thereafter when indicated. The upper reference limit (URL) as defined by the local laboratory was recorded. CK twice the URL was considered evidence of myocardial infarction. Platelet count was also determined at the participating centres. Fibrinogen levels and fibrin(ogen) degradation products, collected at baseline (pretreatment), and at $50 \mathrm{~min}$, and at 12, 24, 48, 96 hours after rt-PA infusion, were determined in a central Coagulation Core Laboratory using standardised methods as described previously. ${ }^{9} 10$

\section{Main outcomes}

The main outcomes of this analysis are the independent association of peak CK activity with adjudicated major bleeding (primary) and with combined adjudicated 
major bleeding, stroke and all-cause mortality during hospitalisation (secondary).

\section{Sample size considerations}

The probability $p$ of events at the mean value of the CK (and other variables) was conservatively estimated to be 0.10 . Based on the previous report, ${ }^{6}$ sample size was estimated for two levels of the OR of disease, corresponding to an increase of one SD from the mean value of $\mathrm{CK}$, given the mean values of the remaining variables; at OR 1.2, with at least 634 participants needed or at OR 2.0, with at least 226 needed for this analysis with a one-tailed alpha of $5 \%$ and 1 -beta of 90 . A second consideration was to include a minimum of 10 events of the least frequent outcome per parameter to fit the model to avoid overestimated or underestimated variances. ${ }^{12}$

\section{Data analyses}

Data of the TIMI 3B study are analysed. Demographic characteristics are reported by bleeding status. Parametric versus non-parametric statistical methods are used where appropriate. CK data are known to be highly skewed to the right, and log transformation to the base of 10 is used in regression analysis. ${ }^{6}$ Mean as well as peak CK during admission are assessed (primary variable), as crude values, and standardised by the local URL. In addition, time to CKmax, and time to severity, and location of the first adjudicated bleeding are noted. Major bleeding is associated with CKmax presented in categories, and as a continuous measure in univariable and multivariable logistic regression analysis, to assess whether log CKmax is independently predictive of fatal or non-fatal major bleeding (primary) or combined major bleeding, stroke and hospital death (secondary), at day 21 after hospital admission. Five covariables are chosen a priori, sex, age, body mass index (BMI, body weight in kilograms divided by the squared height in metres), history of diabetes and systolic blood pressure (SBP) at admission. Other variables are considered based on statistical and clinical criteria and bleeding risk scores ${ }^{1-69-11}$ to find the most parsimonious model that explains the data, using forced entry and bootstrapping for internal validation. Global model fit is based on Bayes information criterion and calibration included Hosmer-Lemeshow statistics. Main subgroup analysis is by rt-PA treatment assignment versus placebo. Sensitivity analysis includes modelling with different relevant covariables and by peak $\mathrm{CK}$ at 24 hours. Model discrimination is assessed with C-statistics. Missing data are not imputed. We limit the use of $p$ values ${ }^{13}$ and communicate statistical uncertainty surrounding estimates through 95\% CIs given between square brackets, with uncertainty regarding the external validity discussed. Data in parentheses are SE unless indicated otherwise. Statistical analyses are performed with the SPSS statistical software package for Windows V.25.0 (SPSS).
RESULTS

\section{Baseline characteristics and assigned treatment}

TIMI 3B patients $(\mathrm{N}=1473)$ were predominantly men $(66.3 \%)$ and white $(79.7 \%)$, with a mean age of 58.8 $(0.3)$ years (table 1$)$. Patients were randomised to rt-PA $(49.4 \%)$ or placebo $(50.6 \%)$ and to the invasive strategy $(49.8 \%)$ versus the conservative strategy $(50.2 \%)$. Median duration of hospitalisation was 11 days (IQR 7-14 days). During the first 5 days of hospitalisation, nearly all patients were given heparin $(99.7 \%)$ and aspirin $(99.5 \%)$, with a median total daily dose (IQR) of 17519 U USP (IQR 13 600-21 320) for heparin and $260 \mathrm{mg}$ (195-284) for aspirin.

\section{Trial outcomes}

TIMI 3 trial endpoints by treatment arm have been reported previously. ${ }^{9}{ }^{10}$ In summary, in TIMI $3 \mathrm{~A}$, of all culprit lesions, $25 \%$ with rt-PA versus $19 \%$ with placebo achieved the primary endpoint of measurable improvement. A thrombus was present in the culprit lesion in 107 patients $(35 \%)$, and in these patients, substantial improvement occurred in $36 \%$ of the patients with rt-PA versus $15 \%$ with placebo. However, in the TIMI 3B study, the incidence of death or non-fatal infarction did not differ after 1 year by strategy assignment, although fewer patients in the early invasive strategy group underwent repeat hospital admission. ${ }^{910}$

\section{Creatine kinase}

CK was estimated at 18 different time points, in total 9215 times in 1470 patients during the hospital stay, from the day of admission to day 17. Plasma CK, missing in four patients, ranged between $<10$ and $19045 \mathrm{IU} / \mathrm{L}$. Mean CK per patient was 221(9) IU/L, with a mean URL of $212 \mathrm{IU} / \mathrm{L}$. CKmax ranged between 15 and $19045 \mathrm{IU} / \mathrm{L}$ (mean 450 (24); median 152, IQR 82-433), a mean of $2.2(0.1)$ times the URL (figure 2A and B). Most patients $(58 \%)$ had a peak CK level below the URL, and only $25 \%$ of the patients had a CK greater than twice normal, with the highest CK activity on the first day in most patients (figure 2C).

\section{Haemorrhagic complications}

Bleeding, reported in 128 patients (8.7\%), was most frequent on the second day after admission (24.2\%, figure 2D). Adjudicated bleeding events were registered in 90 patients, 30 (33.3\%) with major bleeding, 43 (41.1\%) minor bleeding and $23(25.6 \%)$ loss-no-site. Primary bleeding locations are depicted by severity in figure 2E, with surgery-related bleeding (11 patients) classified as 'other'. Stroke (haemorrhagic and non-haemorrhagic) was reported in 18 patients. Thirty patients died during hospitalisation $(2.1 \%)$, of whom $9(30 \%)$ had a major bleeding or stroke. Seven out of 30 patients $(23.3 \%)$ with a major bleeding died, and 64 patients $(4.3 \%)$ had a major bleeding, stroke or hospital death.

Mean CKmax in patients without major bleeding was 439 (23) IU/L, (2.2*URL, SE 0.1), versus 1015 (319) 
IU/L, (5.6*URL, SE 2.4) with major bleeding. The association between CK and major bleeding included 'normal' CK levels (figure $2 \mathrm{~F}$ and $\mathrm{G}$ ). In univariable binary logistic regression analysis, the OR of fatal or non-fatal major bleeding was 3.2 (1.7 to 5.9)/ $/ \log$ CKmax increase. For the composite outcome major bleeding, stroke or hospital death, mean CKmax was 1177 (231), (6.1*URL, SE 1.5); mean age 64 (1.0) years, OR 3.6 (2.3 to 5.7). Sex, age, ancestry (white vs non-white), BMI, a history of diabetes or hypertension, baseline SBP, baseline laboratory values (eg, haematocrit, creatinine, platelet count) and treatment assignment (conservative vs invasive and rt-PA vs placebo) were further considered as predictors of the main primary and secondary outcomes. Data on congestive heart failure and heart rate at admission were not available. Multivariable binary logistic regression analysis suggested an independent association between peak CK and haemorrhagic complications, with per log CK increase a threefold increase in odds for non-fatal or fatal major bleeding and a nearly fourfold increase in combined major bleeding, stroke or hospital death compared with the absence of these outcomes (tables 2 and 3 and figure $2 \mathrm{H}$ ). There was no interaction by sex (data not shown). The C-index of the regression models was 0.8 (figure 3). Although the number of events in the subgroups was too small to build a stable statistical model, the data indicated that the association between CK and major bleeding was present in patients assigned to antithrombotic therapy with added placebo (OR 6.2 (1.7 to 22.4$), \mathrm{n}=703$ ) versus add-on rt-PA (2.3 (1.1 to 4.8 ), $\mathrm{n}=667)$.

\section{DISCUSSION}

To our knowledge, this is the first report on the association of the ADP-scavenging enzyme CK with bleeding during NSTE-ACS. The data indicate that, consonant with earlier findings in STEMI, CK is independently associated with non-fatal and fatal major bleeding, with a three times greater odds compared with the absence of major bleeding and survival, in patients treated with antithrombotic drugs and additional placebo or thrombolytic therapy for NSTE-ACS. The association was independent of heparin dose or rt-PA use and was also observed in patients not receiving thrombolysis. Age was another independent risk factor for major bleeding as previously noted. ${ }^{3}$

The biological plausibility regarding ADP binding (figure 1), the observed strong, consistent, independent association with bleeding in large multicentre clinical studies, the temporal precedence, dose-effect relationship and the experimental evidence on inhibition of platelet aggregation ${ }^{6-8}$ substantiate the hypothesis that CK may increase bleeding risk.

Thrombolytic therapy is no longer indicated in NSTE-ACS management, but plasminogen activators, assigned to half of the presented patients, are still in use for other indications including STEMI and stroke. ${ }^{14}{ }^{15} \mathrm{In}$ addition, current antithrombotic therapy remains strongly associated with major bleeding and subsequent death. ${ }^{1-4}$ In this study, CK was also associated with bleeding in patients assigned to antithrombotic therapy and placebo, as well as in those with additional thrombolytic therapy. We speculate that even at moderately increased levels, CK may act in synergy with drugs that reduce or inhibit platelet aggregation and/or thrombus formation to facilitate major bleeding.

It should be noted that the data of this post hoc analysis were collected in a randomised clinical trial, with patients at high risk for bleeding excluded. A further limitation is that patients were enrolled between 1989 and 1993, with more recent, systematically collected data on CK in patients with ACS lacking. ${ }^{9} 101617$ The 2020 ESC Guidelines for the management of patients with NSTE-ACS ${ }^{1}$ state that: '... it is not recommended to routinely measure additional biomarkers such as CK, CK-MB, h-FABP, or copeptin, in addition to hs-cTn'. However, we propose that because of the strong, proportionate and biologically plausible association of CK with major bleeding observed in NSTE-ACS as well as in STEMI, ${ }^{6}$ plasma CK should be estimated in studies of patients with ACS treated with antiplatelet, anticoagulant or thrombolytic drugs to address the potential association of this enzyme with bleeding. ${ }^{17}$ Presently used risk scores for major bleeding, reporting a C-index of around 0.7 , do not include CK, ${ }^{1-4}$ but it should be studied whether this enzyme could help predict major bleeding and aid in the selection of patients with the best benefit/risk ratio during contemporary ACS treatment.

With the intensification in potency, number of drugs and duration of antithrombotic therapy for ACS, identifying patients at risk for haemorrhagic complications has become a major safety issue. ${ }^{1-4} \mathrm{CK}$, the ADP-binding enzyme that is near to being declared obsolete in diagnostic cardiology, ${ }^{1216}$ might need to be dusted off and reassessed in prospective studies for its potential to contribute to major bleeding during treatment for ACS.

Acknowledgements The manuscript was prepared using TIMI 3 research materials obtained from the NHLBI Biologic Specimen and Data Repository Information Coordinating Center.

Contributors LMB contributed to conceptualisation, data curation, formal analysis, funding acquisition, investigation, methodology, project administration, resources, supervision, validation, visualisation, writing —original draft, writing —review and editing; JF contributed to conceptualisation, investigation, methodology, resources, validation, visualisation, writing — review and editing.

Funding This work is funded by the Dutch CK Science Foundation (national foundation registration number 7106614 ).

Disclaimer The content does not necessarily reflect the opinions or views of the TIMI 3 study members or the NHLBI.

Competing interests LMB is an inventor on patent W0/2012/138226, an 'open' non-restrictive patent request filed and published as 'prior art' to protect the freedom of researchers to operate and share their innovative ideas on CK and CK inhibition without license or payment.

Patient consent for publication Not required.

Ethics approval The study protocol was approved by the Review Board of the Research Ethics Committee of the Radboud University Nijmegen Medical Centre on April 30, 2019 (registration number 2019-5356), and by the US National Heart, Lung, and Blood Institute (RMDA V02 1d20120806) on May 6, 2019. 
Provenance and peer review Not commissioned; externally peer reviewed.

Data availability statement Data are available in a public, open access repository, at the Biologic Specimen and Data Repository Information Coordinating Center (BioLINCC), supported by the National Heart, Lung, and Blood Institute of the United States National Institutions of Health (NIH), USA. https://biolincc.nhlbi.nih. gov/home/.

Open access This is an open access article distributed in accordance with the Creative Commons Attribution Non Commercial (CC BY-NC 4.0) license, which permits others to distribute, remix, adapt, build upon this work non-commercially, and license their derivative works on different terms, provided the original work is properly cited, appropriate credit is given, any changes made indicated, and the use is non-commercial. See: http://creativecommons.org/licenses/by-nc/4.0/.

ORCID iD

Lizzy Maritza Brewster http://orcid.org/0000-0002-7434-0038

\section{REFERENCES}

1 Collet J-P, Thiele H, Barbato E, et al. 2020 ESC guidelines for the management of acute coronary syndromes in patients presenting without persistent ST-segment elevation. Eur Heart J 2020:ehaa575.

2 Levine GN, Bates ER, Bittl JA, et al. 2016 ACC/AHA guideline focused update on duration of dual antiplatelet therapy in patients with coronary artery disease: a report of the American College of Cardiology/American heart association Task force on clinical practice guidelines: an update of the 2011 ACCF/AHA/SCAl guideline for percutaneous coronary intervention, 2011 ACCF/AHA guideline for coronary artery bypass graft surgery, 2012 ACC/AHA/ACP/AATS/ PCNA/SCAI/STS guideline for the diagnosis and management of patients with stable ischemic heart disease, 2013 ACCF/AHA guideline for the management of ST-elevation myocardial infarction, 2014 AHAVACC guideline for the management of patients with non-ST-elevation acute coronary syndromes, and 2014 ACC/ AHA guideline on perioperative cardiovascular evaluation and management of patients undergoing noncardiac surgery. Circulation 2016;134:e123-55.

3 Moscucci M, Fox KAA, Cannon CP, et al. Predictors of major bleeding in acute coronary syndromes: the global registry of acute coronary events (grace). Eur Heart J 2003;24:1815-23.

4 De Silva K, Myat A, Cotton J, et al. Bleeding associated with the management of acute coronary syndromes. Heart 2017;103:546-62.
5 Gremmel T, Frelinger AL, Michelson AD. Platelet physiology. Semin Thromb Hemost 2016;42:191-204.

6 Brewster LM, Fernand J. Creatine kinase is associated with bleeding after myocardial infarction. Open Heart 2020;7:e001261.

7 Brewster LM. Extracellular creatine kinase may modulate purinergic signalling. Purinergic Signal 2020;16:305-12.

8 Brewster LM. Creatine kinase, energy reserve, and hypertension: from bench to bedside. Ann Transl Med 2018;6:292.

9 Investigators TI. Early effects of tissue-type plasminogen activator added to conventional therapy on the culprit coronary lesion in patients presenting with ischemic cardiac pain at rest. Results of the thrombolysis in myocardial ischemia (TIMI IIIA) trial. Circulation 1993;87:38-52.

10 Investigators TI. Effects of tissue plasminogen activator and a comparison of early invasive and conservative strategies in unstable angina and non-Q-wave myocardial infarction. Results of the TIMI IIIB trial. thrombolysis in myocardial ischemia. Circulation 1994;89:1545-56.

11 Bovill EG, Terrin ML, Stump DC, et al. Hemorrhagic events during therapy with recombinant tissue-type plasminogen activator, heparin, and aspirin for acute myocardial infarction. Results of the thrombolysis in myocardial infarction (TIMI), phase II trial. Ann Intern Med 1991;115:256-65.

12 Hosmer DW, Lemeshow S. Ch. 8.5: Sample size issues when fitting logistic regression models. In: Applied logistic regression. 2nd Ed. New York: John Wiley and Sons Inc, 2000: 346-7.

13 Wasserstein RL, Schirm AL, Lazar NA. Moving to a world beyond "p<0.05". Am Stat 2019;73:1-19.

14 McCaul M, Lourens A, Kredo T. Pre-Hospital versus in-hospital thrombolysis for ST-elevation myocardial infarction. Cochrane Database Syst Rev 2014:CD010191.

15 Powers WJ, Rabinstein AA, Ackerson T, et al. 2018 guidelines for the early management of patients with acute ischemic stroke: a guideline for healthcare professionals from the American heart Association/ American stroke association. Stroke 2018;49:e46-110.

16 Alvin MD, Jaffe AS, Ziegelstein RC, et al. Eliminating creatine kinase-myocardial band testing in suspected acute coronary syndrome: a value-based quality improvement. JAMA Intern Med 2017;177:1508-12.

17 Brewster LM. Because of its association with major bleeding the ADP-binding enzyme creatine kinase should be estimated in studies of patients treated for non-ST-segment elevation acute coronary syndromes (NSTE-ACS). Letter. Eur Heart J 2020: ehaa905. 